\title{
Kinesin inhibitor marches toward first-in-class pivotal trial
}

The key to treating cancer is to put a stop to the out-of-control cell growth that leads to tumor formation. One way to do this is to go after the microtubules that help coordinate this rampant cell division. Yet because microtubules function in both dividing and non-dividing cells-for example, in non-dividing neurons they're involved in intracellular transportdrugs that target microtubules directly tend to cause nerve pain and other side effects. That's why researchers have been on the lookout for more specific targets in the microtubule machinery-ones that are only active in rapidly growing cells during mitosis.

The kinesin spindle protein (KSP), a molecular motor that crawls along the microtubules to help the cells divide, provides one such candidate target. To date, drugs designed to block this protein (which is also known as Eg5) have failed to live up to their potential, with something of a KSP inhibitor graveyard littered with failed and abandoned products from companies including Cytokinetics, AstraZeneca, Eli Lilly and others. The drug companies "were getting the effect on the chromosomes they wanted, but the cells were not dying as they were supposed to," says Charles Saxe, director of the cell biology and metastasis program at the American Cancer Society in Atlanta, who was not involved in the development of any of these products.

Now, in a first for the field, a KSP-targeted drug looks set to enter late-stage clinical testing. On 31 October, Array BioPharma of Boulder, Colorado, announced plans to launch a global phase 3 trial for its KSP inhibitor, ARRY-520. That study, which is slated to begin toward the middle of next year, will pair ARRY520 with a proteasome inhibitor called Kyprolis (carfilzomib) in several hundred people with relapsed or refractory multiple myeloma. In an earlier 20-person dose-escalation trial, $37 \%$ of participants who received ARRY520 and Kyprolis showed a reduction in the harmful monoclonal 'M' protein produced by multiple myeloma cells, compared with $16 \%$ of historical controls who received Kyprolis alone.

The results of this study will be presented on 7 December at the American Society of Hematology (ASH) Annual Meeting in New Orleans. Additional data will also be described at the conference involving the use of ARRY520 as a monotherapy or given together with the steroid drug dexamethasone and Velcade (bortezomib), another proteasome inhibitor.

Kevin Koch, president and chief scientific officer of Array, is confident that ARRY-520 will avoid the pitfalls of its predecessors. "There

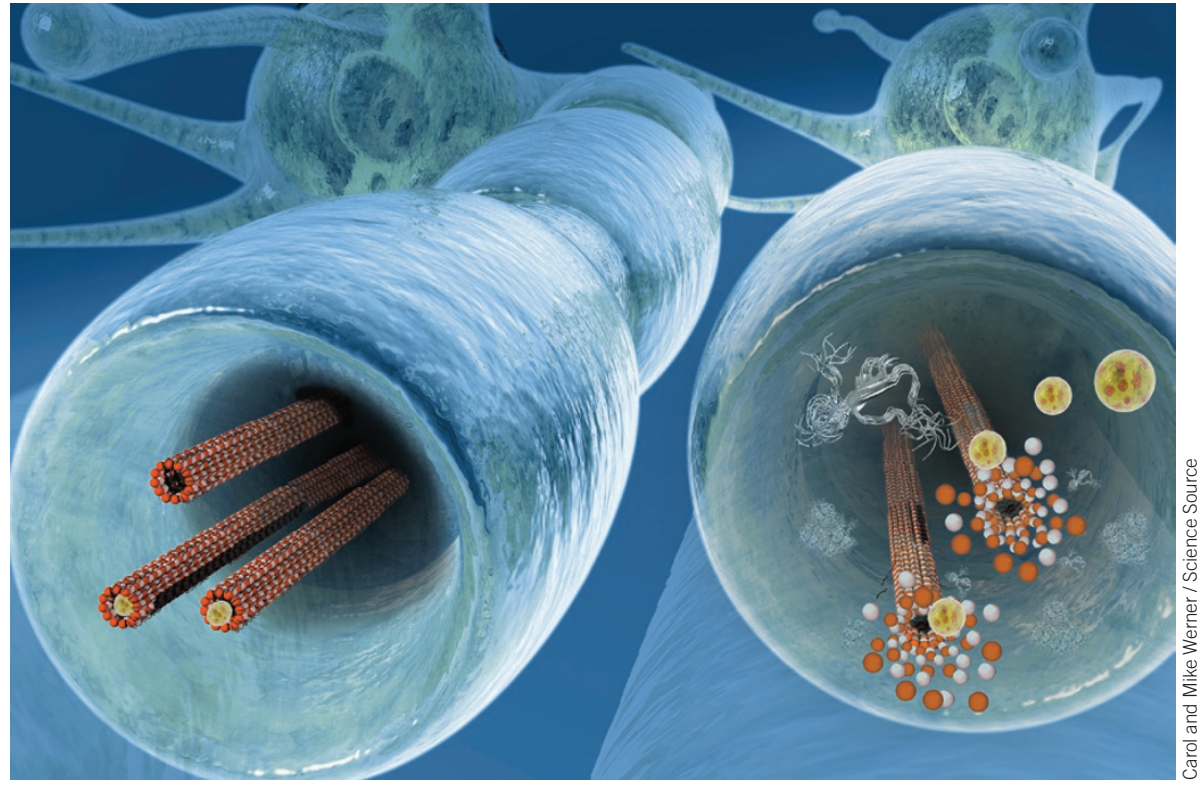

Spindle doctor: Targeting the microtubule motor protein KSP is finally showing clinical promise.

are a couple of things that are unique to the molecule," he says. For one, the drug tends to be active in cell lines that are resistant to other front-line chemotherapy agents such as chemotherapeutic taxanes, which attack microtubules directly. Moreover, ARRY-520 also seems to inhibit the proteins responsible for regulating cell death, making it easier for the drug to kill cancer cells.

\section{Marker of response}

Array is also looking at a potential biomarker, alpha-1 acidic glycoprotein (AAG), which is often elevated in people with multiple myeloma. AAG is a 'cleanup' protein in the blood serum that can bind to ARRY-520, rendering it ineffective. In a 32-person trial in which patients with myeloma received ARRY-520 alone, 5 of the 21 participants with low AAG levels showed a reduction in $\mathrm{M}$ protein that lasted for more than 8 months on average, whereas none of the 5 participants with high AAG levels experienced such a favorable response.

According to Koch, the findings, which will also be presented at the December ASH meeting, suggest that AAG levels could help indicate who might be a good candidate for the drug. "There are very few mechanisms that show such strong single-agent activity," he says.

Array is not the only company that has faith in the KSP pathway. At least two other companies, ArQule and Alnylam Pharmaceuticals, both based in the Boston area, are developing compounds that target
KSP. ArQule's ARQ 621 is being tested in both hematological and solid tumor cancers, while Alnylam's ALN-VSP is focused on liver cancer. ALN-VSP is unique among these drugs, as it does not target the KSP protein directly, but uses RNA interference to prevent it from being expressed in the first place. (ARQ 621, like ARRY-520, is a typical small molecule that's administered intravenously.) So far, both have only been tested in phase 1 trials.

At first, ARRY-500 will probably be used only in people with myeloma who have failed on other drugs. But Andy Robbins, Array's senior vice-president of commercial and strategy, hopes that KSP inhibitors like ARRY-520 will one day form a frontline therapy, alongside the two entrenched classes of therapies: immunomodulators such as Revlimid (lenalidomide) and proteosome inhibitors such as Kyprolis and Velcade. "We have the potential to be a third backbone treatment," he says.

Brian Owens

\section{Correction}

In the November 2013 issue, the article entitled "Trial designs advance to overcome bitter pill of placebo effect" (Nat. Med. 19, 1353,2013 ) incorrectly quoted Richard Pops as saying that SPCD trials enable power with smaller ends, when it should have said a smaller number of subjects. The error has been corrected in the HTML and PDF versions of the article. 